\title{
Relationship between diabetes risk and admixture in postmenopausal African-American and Hispanic-American women
}

\author{
L. Qi • R. Nassir • R. Kosoy • L. Garcia • J. D. Curb • \\ L. Tinker • B. V. Howard • J. Robbins • M. F. Seldin
}

Received: 14 September 2011 / Accepted: 11 January 2012 / Published online: 10 February 2012

(C) Springer-Verlag 2012

\begin{abstract}
Aims/hypothesis Type 2 diabetes is more prevalent in AfricanAmericans (AFAs) and Hispanic-Americans (HAs) than in European-Americans. We assessed whether continental admixture was correlated with diabetes risk in these high-risk groups. Methods We estimated the proportion of sub-Saharan African (AFR), Amerindian (AMI) and European admixture using 92 ancestry-informative marker genotypes in 16,476 AFA and HA women from the Women's Health Initiative. Cox regression models were used to examine the association between admixture and diabetes risk, with and without accounting for socioeconomic status (SES) and adiposity measurements.

Results AFR admixture was significantly associated with diabetes risk in AFA women when adjusting for entry age,
\end{abstract}

Electronic supplementary material The online version of this article (doi:10.1007/s00125-012-2486-4) contains peer-reviewed but unedited supplementary material, which is available to authorised users.

L. Qi $\cdot$ L. Garcia

Department of Public Health Sciences, University of California,

Davis, CA, USA

R. Nassir • R. Kosoy $\cdot$ J. Robbins $\cdot$ M. F. Seldin $(\triangle)$

Department of Biochemistry and Molecular Medicine,

University of California, UC Davis,

4453 Tupper Hall, One Shields Ave,

Davis, CA 95616, USA

e-mail: mfseldin@ucdavis.edu

R. Nassir $\cdot$ R. Kosoy $\cdot$ J. Robbins $\cdot$ M. F. Seldin

Department of Internal Medicine, University of California,

Davis, CA, USA

J. D. Curb

Department of Geriatric and Internal Medicine, John A Burns

School of Medicine, University of Hawaii, Manoa,

Honolulu, HI, USA neighbourhood SES and BMI or waist/hip ratio (WHR) (all $p<0.0001$ ). In HA women, AMI admixture had significant associations with diabetes risk that remained significant after adjustment for SES and BMI (all $p<0.0005$ ). In both AFAs and HAs, SES showed significant negative associations while BMI or WHR had significant positive associations with diabetes risk, with and without adjustment for genetic admixture.

Conclusions/interpretation In AFAs, admixture, SES and BMI/WHR each independently contribute to diabetes risk after accounting for each of the other factors; in HAs, admixture, SES and BMI each independently contribute to diabetes risk after accounting for each of the other factors, whereas admixture is not significantly associated with diabetes risk

L. Tinker

Fred Hutchinson Cancer Research Center, Seattle, WA, USA

B. V. Howard

MedStar Health Research Institute, Hyattsville, MD, USA

B. V. Howard

Georgetown and Howard Universities Center

for Clinical and Translational Science,

Washington, DC, WA, USA 
after accounting for SES and WHR. The findings emphasise the importance of considering both genetic and environmental causes in the aetiology of type 2 diabetes.

Keywords Body mass index · Diabetes · Ethnicity . Genetics $\cdot$ Waist/hip circumference ratio

$\begin{array}{ll}\text { Abbreviations } \\ \text { AFA } & \text { African-American } \\ \text { AFR } & \text { Sub-Saharan African } \\ \text { AIMs } & \text { Ancestry informative markers } \\ \text { AMI } & \text { Amerindian } \\ \text { CEU } & \text { Centre d'Etude du Polymorphisme } \\ & \text { Humain Europeans } \\ \text { EUR } & \text { European } \\ \text { EA } & \text { European-American } \\ \text { HA } & \text { Hispanic-American } \\ \text { SES } & \text { Socioeconomic status } \\ \text { SNP } & \text { Single nucleotide polymorphism } \\ \text { WHI } & \text { Women's Health Initiative }\end{array}$

\section{Introduction}

Diabetes has reached worldwide epidemic levels in virtually all ethnic groups, but is proportionately of higher frequency in many non-European populations [1,2]. In the USA, the ratio of diabetes in African-Americans (AFAs) compared with European-Americans (EAs) is 1.6 to 2.0 [3]. Similarly, the age-adjusted prevalence of diabetes has been estimated as $13.3 \%$ for Mexican-Americans and $13.8 \%$ for Puerto Ricans compared with $7.1 \%$ for non-Hispanic whites [4]. The age-adjusted prevalence for women showed a similar disparity between these ethnic groups: 9.1\% for MexicanAmericans and 8.4\% for Puerto Ricans compared with 5.1\% for non-Hispanic whites [3].

These differences in prevalence suggest that diabetes risk factors, including both genetic and/or environmental components, occur more frequently in these admixed ethnic groups. In the USA, the AFA population is typically an admixture of European (EUR) and sub-Saharan African (AFR) ancestry in different proportions [5-9]; and the Hispanic-American (HA) population generally shows a multi-way admixture with varying proportions of ancestral EUR, Amerindian (AMI), and AFR contributions [10-13]. Several studies of HA populations demonstrate or suggest associations between type 2 diabetes and individual AMI ancestry proportions [14-17]. However, these studies have examined relatively small participant sets $(<1000$ participants) and have reported substantial confounding by socioeconomic factors. For AFA populations, although several studies have examined the correlation between AFR (or EUR) admixture and adiposity measures [5, 8, 18, 19], investigations on the admixture-diabetes association have been limited. To our knowledge, only one study addressed this issue and reported that a higher AFR admixture was observed in AFAs with diabetic end-stage renal disease [20].

As well as genetic factors, socioeconomic effects including poverty, education and lack of access to care have been implicated as major factors in differences in diabetes prevalence in AFAs and EAs [21-24]. A recent study showed both individual and neighbourhood socioeconomic status (SES) to be associated with diabetes risk in AFA women [25]. However, the relative effect of SES varies greatly between studies [21-24, $26,27]$. Differences in diabetes prevalence are also attributed to differences in BMI, and both BMI and waist-to-hipcircumference ratio (WHR) have been used to assess the impact of adiposity and/or fat distribution on diabetes and other health consequences [28-31]. Potential differences between the importance of BMI and WHR in different population groups are also suggested by some previous studies [19, 29, 31].

As another step in assessing potential differences in diabetes risk, we investigated the relationship between diabetes risk and the relative proportion of AFR, AMI and EUR admixture in the Women's Health Initiative (WHI) participants who selfidentified as AFA or HA. We used a set of ancestry informative markers (AIMs) that enable the accurate estimation of AFR, AMI and EUR admixture proportions in AFAs and HAs $[19,32,33]$. In addition, we also investigated whether SES, BMI and WHR influence the admixture associated risks for diabetes in this large study.

\section{Methods}

Study participants The WHI is the largest US health study in a multiethnic group of postmenopausal women aged 5079 years, and consists of four randomised controlled clinical trials and an observational study. This study involves 40 clinical centres and recruited over 160,000 women between 1993 and 1998. Detailed descriptions of the WHI are available [34-36]. The current study included 16,476 self-identified AFA and HA WHI women for whom both quality-filter genotypes [19] and diabetes information were available. The study was conducted with appropriated informed consent and in agreement with established Human Institutional Review Board procedures.

Outcomes We considered a time-to-event outcome which was time-to-diabetes for diabetic participants and censoring time for non-diabetic participants as of September 2005. A participant was diabetic if she had been treated for diabetes at baseline or during follow-up as of September 2005. A woman had diabetes at baseline if she reported at study entry that she had received a physician diagnosis of sugar diabetes when not pregnant and received glucose-lowering medications. 
Age range when a participant was first told to have nonpregnancy-related diabetes was reported as <21, 21-29, 30$39,40-49,50-59,60-69$ or 70 years or older. We used the middle point of the corresponding age ranges (e.g. 45 for the range 40-49) as time to event for the participants with baseline diabetes. Participants had diabetes during follow-up if they reported a new physician diagnosis of diabetes treated with glucose-lowering agents. Assuming birth as time 0 , time to diabetes was considered as the event time for participants with diabetes, and for participants who did not have diabetes as of September 2005, their last time of follow-up or death time was the censoring time.

AIMs The AIMs were chosen based on our previous studies $[32,33]$. The final marker set included 92 single-nucleotide polymorphisms (SNPs) that demonstrated large differences in allele frequency between ancestral populations from Europe, sub-Saharan Africa and the Americas. This SNP set shows a mean $90 \%$ Bayesian confidence limit of 0.2 for admixture assessment in our studies of groups of AFAs, MexicanAmericans and Puerto Ricans [32]. While the mean confidence limits are larger in smaller AIM sets compared with larger sets of AIMs (the mean $90 \%$ confidence limits $\sim 0.1$ for sets containing $>1,500$ AIMs), the admixture proportions estimated in individuals assessed with these highly selected AIMs [32] are strongly correlated with larger AIMs sets. Correlations with larger sets of SNP AIMs ( $>500$ SNPs) are $>0.9$ in testing with tested Mexican-American and AFA admixed populations (mean difference in admixture fraction $<0.05$ ) (M. F. Seldin, unpublished data).

Genotyping and admixture assessment Genotyping was performed as previously described [19] using the TaqMan OpenArrays system (Life Technologies/Applied Biosystems, Foster City, CA, USA). The mean call rate for the included women was 97.1\%. All AIM SNPs were in HardyWeinberg equilibrium $(p>0.005)$ in parental populations.

AFR, AMI and EUR contribution to each self-identified AFA or HA woman was examined using STRUCTURE analyses of genotyping results with AIMs as previously described $[32,33]$. We considered three groups separately: All (all selfidentified AFA and HA participants); whole AFA (all selfidentified AFA participants); and HA (self-identified HA participants). For each group, representatives of three parental populations (AFR, AMI and EUR) were considered for analyses under the assumption of three populations $(K=3)$. For whole AFA, to minimise potential database or laboratory errors that cause miss-assignment, we further excluded extreme outliers and re-evaluated the subgroup with $>20 \%$ AFR and $<5 \%$ AMI admixture under the assumption of two parental populations $(K=2)$ where the contribution of AFR and EUR was considered. The samples used to represent the parental population groups included: 128 European-Americans from the New York Cancer Project and 60 Centre d'Etude du Polymorphisme Humain Europeans (CEU) for EUR; 56 Yoruban African, 19 Bini West African and 23 Kanuri West African for AFR; and 50 Mayan Amerindians, 26 Quechuan Amerindians and 29 Nahua Amerindians for AMI as previously described [32]. For each analysis, we conducted three independent runs using STRUCTURE v2.3.3 [37, 38] and prior parental population assignment, with 100,000 replicates and 100,000 burn-in cycles. The results were consistent with $<0.02$ difference between each of the independent runs.

SES and other covariates SES was obtained using a standardised geocoding protocol [39, 40], which linked individual WHI observational study and clinical trial participant addresses to year 2000 US Census Federal Information Processing Standards (FIPS) codes and tract-level socioeconomic data. A summary measure of each participant's neighbourhood socioeconomic environment was estimated from the tractlevel data using six variables representing several dimensions of wealth and income: (1) natural log of median household income; (2) natural log of median value of housing units; (3) percentage of households receiving interest, dividend or net rental income; (4) percentage of adults $>25$ years of age who had completed high school; (5) percentage of adults $>25$ years of age who had completed college; and (6) percentage of employed persons $>16$ years of age in executive, managerial or professional specialty occupations. The six variables were converted into standardised $(z)$ scores by subtracting the population-specific mean from the value associated with each participant's census tract and then dividing the difference by the population-specific SD. The transformation was performed separately within the observational study and clinical trial and generated six $z$ scores, each of which indicated the deviation of the tract-level value from the corresponding population-specific mean and summed to 0 across the population. A neighbourhood summary $z$ score was then constructed by summing the six $z$ scores. As we aimed at controlling for neighbourhood SES among combined populations of both the observational study and clinical trial participants we included an indicator for study cohort in the regression analyses.

Entry age, BMI and WHR were determined from baseline WHI data. Weight was measured to the nearest $0.1 \mathrm{~kg}$ on a balance beam scale. Height was measured to the nearest $0.1 \mathrm{~cm}$ using a wall-mounted stadiometer. Waist and hip circumferences were measured at baseline using standard tape measures. BMI was calculated as weight $(\mathrm{kg})$ divided by the square of measured height $\left(\mathrm{m}^{2}\right)$. WHR was computed as the ratio of waist circumference $(\mathrm{cm})$ to hip circumference $(\mathrm{cm})$.

Statistical analysis Analyses were performed separately in the following groups: All (all self-identified AFA and HA participants), AFA (self-identified AFA participants with $>20 \%$ AFR 
and $<5 \%$ AMI admixture) and HA (self-identified HA participants). Descriptive statistics are provided in Table 1. As SES was calculated separately for observational study and clinical trial participants, the values of SES were normalised to be between 0 and 1 for descriptive statistics. Two sample $t$ tests were used to assess the mean differences of these variables between the two groups of participants. Pearson correlation coefficients were obtained to examine the pairwise correlation between each of the admixture proportion, SES, BMI and WHR.

HRs and $95 \%$ CIs for diabetes risk in relation to admixture were estimated using Cox regression models [41] in which time-to-diabetes or time-to-censoring was the outcome variable. The HR is the ratio of the hazard rates of two groups with one unit difference in the independent variable and is estimated by the exponential of the coefficient estimate of this variable in the model. For All and HA groups, AFR, EUR and AMI admixtures were considered in separate models; for the AFA group, only AFR admixture was considered because this group only contained AFR and EUR admixtures, and the results for EUR mirror those for AFR. We considered models adjusting for: (1) entry age alone; and (2) entry age, SES and the indicator variable for population (clinical trial or observational study). We conducted analyses with and without controlling for BMI or WHR in the Cox model as adiposity measures are expected to be intermediate outcomes of admixture and thus inclusion of these measures in the Cox regression models may diminish the admixture-diabetes association. For all Cox models, SES, BMI and WHR were standardised to one SD based on the distribution of each group (All, AFA and HA) for analysis. This method enables direct comparison of estimates across these variables, and the estimated HRs correspond to the effects of one SD change in the variables.

We also investigated the associations between SES and diabetes risk with and without controlling for admixture, and the associations between BMI (and WHR) and diabetes risk with and without including SES and admixture in the Cox models. All these models include entry age. We estimated the percentage of explained randomness, analogous to explained variation for the Cox model, according to O'Quigley et al. [42].

A sensitivity analysis was performed to assess the amount of loss of information associated with using the midpoint of the age ranges for women who had diabetes at baseline. Almost identical results to those that used the midpoint were obtained when, for each of these women, a randomly chosen value from her age range was used as time to diabetes, suggesting minimal loss of information associated with using the midpoint.

Analyses were carried out using SAS version 9.2 (SAS Institute, Cary, NC USA) and R (www.r-project.org/). All statistical tests were two-sided and $p<0.05$ was considered statistically significant.

\section{Results}

Descriptive statistics of entry age, BMI, WHR and SES Among 16,476 AFA and HA women, 3,439 women had diabetes by September 2005. The average age of first occurrence of diabetes was 58.9 years $(\mathrm{SEM}=0.19)$ for the diabetic women.
Table 1 Descriptive statistics of variables by diabetes status

\footnotetext{
${ }^{a}$ The groups included all selfidentified AFAs and HAs (All), self-identified AFAs with $>0.2$ AFR admixture and $<0.05$ AMI (AFA) and self-identified HAs (HA)

${ }^{\mathrm{b}} p$ values were obtained from two-sample $t$ tests

${ }^{\mathrm{c}} \mathrm{SES}$ normalised to have values between 0 and 1
}

\begin{tabular}{|c|c|c|c|c|c|c|c|}
\hline \multirow[t]{2}{*}{ Group $^{a}$} & \multicolumn{3}{|l|}{ Non-diabetic } & \multicolumn{3}{|l|}{ Diabetic } & \multirow[t]{2}{*}{$p$ value $^{\mathrm{b}}$} \\
\hline & Sample size & Mean & SEM & Sample size & Mean & SEM & \\
\hline \multicolumn{8}{|c|}{ Entry age } \\
\hline All & 13,037 & 61.0 & 0.062 & 3,439 & 61.8 & 0.117 & $2.0 \times 10^{-8}$ \\
\hline AFA & 8,238 & 61.5 & 0.079 & 2,415 & 62.2 & 0.140 & $3.4 \times 10^{-5}$ \\
\hline HA & 4,169 & 60.1 & 0.105 & 811 & 60.7 & 0.238 & $2.2 \times 10^{-2}$ \\
\hline \multicolumn{8}{|l|}{ BMI } \\
\hline All & 13,037 & 29.8 & 0.053 & 3,439 & 32.9 & 0.112 & $5.8 \times 10^{-134}$ \\
\hline AFA & 8,238 & 30.4 & 0.069 & 2,415 & 33.4 & 0.136 & $1.2 \times 10^{-78}$ \\
\hline HA & 4,169 & 28.4 & 0.082 & 811 & 31.6 & 0.211 & $7.1 \times 10^{-42}$ \\
\hline \multicolumn{8}{|l|}{ WHR } \\
\hline All & 13,037 & 0.81 & 0.001 & 3,439 & 0.86 & 0.001 & $3.5 \times 10^{-242}$ \\
\hline AFA & 8,238 & 0.81 & 0.001 & 2,415 & 0.86 & 0.002 & $1.2 \times 10^{-157}$ \\
\hline HA & 4,169 & 0.81 & 0.001 & 811 & 0.86 & 0.003 & $1.5 \times 10^{-73}$ \\
\hline \multicolumn{8}{|l|}{$\mathrm{SES}^{\mathrm{c}}$} \\
\hline All & 13,037 & 0.59 & 0.001 & 3,439 & 0.55 & 0.002 & $1.9 \times 10^{-50}$ \\
\hline AFA & 8,238 & 0.58 & 0.001 & 2,415 & 0.55 & 0.002 & $5.7 \times 10^{-23}$ \\
\hline HA & 4,169 & 0.60 & 0.002 & 811 & 0.55 & 0.005 & $5.6 \times 10^{-24}$ \\
\hline
\end{tabular}


For non-diabetic women, the average age at last follow-up or death was 68.5 years $(\mathrm{SEM}=0.06)$. Table 1 presents the summary statistics by diabetes status for the study participants. Admixture proportions are not included in the summary data in compliance with the current WHI policy. The WHI rationale for the policy is to avoid release of information that is viewed as sensitive by many participants and was not specifically addressed in the consent form. In each group, entry age, BMI and WHR were significantly higher among the diabetic participants than among the non-diabetic participants $(p<0.0001)$ while SES was significantly lower among the diabetic participants than among the non-diabetic participants $(p<0.0001)$. For AFA and HA women, pairwise correlations were also obtained between each of the admixture proportions, SES, BMI and WHR (electronic supplementary material [ESM] Table 1). In AFAs, SES had negative correlations with AFR, BMI and WHR; AFR had positive correlations with BMI and WHR and its correlation with BMI was stronger than that with WHR. In HAs, SES had negative correlations with AFR, AMI, BMI and WHR while it had a positive correlation with EUR; EUR also had negative correlations with BMI and WHR while AMI had positive correlations with BMI and WHR, and its correlation with WHR was stronger than that with BMI. All correlations were significant $(p<0.05)$ except for the correlations between AFR and BMI/WHR in HAs.

Association between admixture and diabetes risk Cox regression analyses showed significant associations of diabetes risk and admixture (Table 2). In All, where combined AFA and HA participants were considered, AFR admixture was positively associated with diabetes risk when adjusting for entry age (HR 1.82, $p<0.0001)$. This positive association remained when further controlling for SES, and when including BMI or WHR in the models $(p<0.0001)$. In contrast, EUR admixture was negatively associated with diabetes risk in all the models $(p<0.0001)$, indicating protective effects against diabetes. AMI admixture was significantly associated with diabetes risk in all models except in the model including entry age, SES and BMI.

In the AFA group, a higher proportion of AFR admixture increased the diabetes risk when adjusting for entry age (HR $3.08, p<0.0001)$. After further adjusting for SES, this positive admixture-diabetes association remained highly significant albeit with a smaller HR, 2.36. The HR decreased modestly when BMI or WHR was also included in the Cox model but remained significant $(p<0.0001)$.

In HAs, where most participants had a substantial proportion of AMI admixture, AFR admixture was not significantly associated with diabetes risk. However, AMI admixture showed a significant positive association with diabetes risk, which remained significant after adjusting for entry age alone, or with SES, or with SES and BMI. However, the association
Table 2 Survival analysis of the association of admixture with diabetes risk

\begin{tabular}{|c|c|c|c|c|c|}
\hline Group $^{a}$ & Admixture $^{\mathrm{b}}$ & Model & HR & $95 \% \mathrm{CI}$ & $p$ value \\
\hline \multirow[t]{12}{*}{ All } & AFR & Age & 1.82 & $1.63,2.04$ & $3.2 \times 10^{-25}$ \\
\hline & AFR & Age, SES & 1.64 & $1.46,1.83$ & $1.7 \times 10^{-17}$ \\
\hline & AFR & Age, SES, BMI & 1.33 & $1.19,1.50$ & $9.8 \times 10^{-7}$ \\
\hline & AFR & Age, SES, WHR & 1.58 & $1.41,1.77$ & $2.0 \times 10^{-15}$ \\
\hline & EUR & Age & 0.39 & $0.33,0.45$ & $1.3 \times 10^{-32}$ \\
\hline & EUR & Age, SES & 0.46 & $0.39,0.54$ & $8.9 \times 10^{-22}$ \\
\hline & EUR & Age, SES, BMI & 0.59 & $0.50,0.69$ & $9.0 \times 10^{-11}$ \\
\hline & EUR & Age, SES, WHR & 0.50 & $0.43,0.59$ & $3.7 \times 10^{-17}$ \\
\hline & AMI & Age & 0.69 & $0.55,0.88$ & $1.6 \times 10^{-3}$ \\
\hline & AMI & Age, SES & 0.70 & $0.55,0.88$ & $2.3 \times 10^{-3}$ \\
\hline & AMI & Age, SES, BMI & 0.97 & $0.77,1.23$ & $8.2 \times 10^{-1}$ \\
\hline & AMI & Age, SES, WHR & 0.64 & $0.50,0.80$ & $1.3 \times 10^{-4}$ \\
\hline \multirow[t]{4}{*}{ AFA } & AFR & Age & 3.08 & $2.24,4.24$ & $4.8 \times 10^{-12}$ \\
\hline & AFR & Age, SES & 2.36 & $1.71,3.27$ & $2.3 \times 10^{-7}$ \\
\hline & AFR & Age, SES, BMI & 2.02 & $1.46,2.80$ & $2.2 \times 10^{-5}$ \\
\hline & AFR & Age, SES, WHR & 2.23 & $1.61,3.09$ & $1.5 \times 10^{-6}$ \\
\hline \multirow[t]{12}{*}{ HA } & AFR & Age & 1.11 & $0.64,1.92$ & $7.2 \times 10^{-1}$ \\
\hline & AFR & Age, SES & 0.91 & $0.53,1.59$ & $7.5 \times 10^{-1}$ \\
\hline & AFR & Age, SES, BMI & 0.84 & $0.48,1.48$ & $5.6 \times 10^{-1}$ \\
\hline & AFR & Age, SES, WHR & 0.98 & $0.57,1.68$ & $9.3 \times 10^{-1}$ \\
\hline & EUR & Age & 0.40 & $0.28,0.57$ & $5.1 \times 10^{-7}$ \\
\hline & EUR & Age, SES & 0.54 & $0.37,0.77$ & $8.4 \times 10^{-4}$ \\
\hline & EUR & Age, SES, BMI & 0.54 & $0.37,0.78$ & $1.0 \times 10^{-3}$ \\
\hline & EUR & Age, SES, WHR & 0.71 & $0.49,1.04$ & $7.7 \times 10^{-2}$ \\
\hline & AMI & Age & 2.50 & $1.74,3.60$ & $8.8 \times 10^{-7}$ \\
\hline & AMI & Age, SES & 1.97 & $1.36,2.84$ & $3.1 \times 10^{-4}$ \\
\hline & AMI & Age, SES, BMI & 2.03 & $1.40,2.95$ & $1.9 \times 10^{-4}$ \\
\hline & AMI & Age, SES, WHR & 1.42 & $0.97,2.06$ & $6.7 \times 10^{-2}$ \\
\hline
\end{tabular}

SES, BMI and WHR were divided by their SDs

${ }^{\text {a }}$ Groups are defined as noted in the footnote to Table 1

${ }^{\mathrm{b}}$ The AFR, AMI or EUR admixture was used as the covariate of interest in separate models, adjusting for the variables listed in each model: age (entry age), SES and BMI or WHR

between diabetes risk and AMI admixture became nonsignificant (HR 1.42, $p=0.067$ ) when WHR was included in the Cox model together with entry age and SES, suggesting that WHR may be a mediator for diabetes among the HA participants.

We also conducted analyses adjusting for whether a woman was in the observational study or clinical trial arm of any WHI clinical trials (data not shown) or excluding women in an active clinical trial treatment arm. The results were very similar (ESM Table 2).

Association between SES and diabetes risk adjusting for admixture SES had a significant negative association with diabetes risk when adjusting for entry age (HRs range from 
0.69 to $0.82, p<0.0001$ ) (Table 3 ). This association remained significant when further adjusting for admixture in all three groups (HRs range from 0.69 to $0.84, p<0.0001$ ), suggesting that SES has an independent contribution to diabetes risk.

Association between BMI/WHR and diabetes risk adjusting for admixture Both BMI and WHR were significantly associated with diabetes risk when adjusting for entry age in the All, AFA and HA groups (HRs range from 1.46 to 1.61 for BMI, and 1.64 to 1.68 for WHR, $p<0.0001$ ) (Table 4). These associations were similar when further adjusting for admixture and SES in all three groups (HRs range from 1.42 to 1.56 for BMI and 1.63 to 1.69 for WHR in various models, $p<0.0001$ ), suggesting an independent contribution of BMI or WHR to diabetes risk in these groups.

\section{Discussion}

In this study, we show an association between type 2 diabetes and AFR admixture proportion in AFA women, with and without taking SES and/or adiposity measures (BMI or WHR) into account. Our results showing a positive association between AFR admixture and diabetes risk are consistent with findings from a recent study of AFA women with diabetes and end-stage renal disease [20]. Importantly, our study features a much larger sample size, is restricted to adult postmenopausal women, is not limited to diabetic individuals with end-stage renal disease and includes a measure of SES. We also found that AMI admixture had significant positive associations with diabetes risk in HA women, with and without adjusting for SES and/or BMI. A study of 561 men and women in Mexico City also reported a

Table 3 Survival analysis of the association of SES with diabetes risk

\begin{tabular}{lllll}
\hline Group $^{\text {a }}$ & Model & HR & $95 \%$ CI & $p$ value \\
\hline All & Age & 0.78 & $0.75,0.80$ & $2.9 \times 10^{-46}$ \\
& Age, AFR & 0.79 & $0.76,0.81$ & $7.8 \times 10^{-39}$ \\
& Age, EUR & 0.80 & $0.77,0.83$ & $3.1 \times 10^{-35}$ \\
& Age, AMI & 0.77 & $0.75,0.80$ & $3.1 \times 10^{-46}$ \\
AFA & Age & 0.82 & $0.79,0.85$ & $2.8 \times 10^{-21}$ \\
& Age, AFR & 0.84 & $0.80,0.87$ & $1.4 \times 10^{-16}$ \\
HA & Age & 0.69 & $0.65,0.74$ & $1.2 \times 10^{-23}$ \\
& Age, AFR & 0.69 & $0.64,0.74$ & $1.3 \times 10^{-23}$ \\
& Age, EUR & 0.71 & $0.66,0.76$ & $1.2 \times 10^{-20}$ \\
& Age, AMI & 0.70 & $0.66,0.76$ & $3.0 \times 10^{-21}$ \\
\hline
\end{tabular}

SES was used as the covariate of interest in the models, adjusting for age (entry age), and each of the genetic admixtures: AFR, EUR or AMI separately

SES was divided by its SD

${ }^{\text {a }}$ Groups are defined as noted in the footnote to Table 1
Table 4 Survival analysis of the association of BMI and WHR with diabetes risk

\begin{tabular}{|c|c|c|c|c|c|}
\hline \multirow[t]{2}{*}{ Group $^{a}$} & \multirow[t]{2}{*}{ Model } & \multicolumn{2}{|c|}{$\mathrm{BMI}^{\mathrm{b}}$} & \multicolumn{2}{|c|}{$\mathrm{WHR}^{\mathrm{b}}$} \\
\hline & & $\mathrm{HR}$ & $95 \% \mathrm{CI}$ & HR & $95 \% \mathrm{CI}$ \\
\hline \multirow[t]{4}{*}{ All } & Age & 1.50 & $1.46,1.55$ & 1.68 & $1.63,1.72$ \\
\hline & Age, SES, AFR & 1.45 & $1.40,1.49$ & 1.67 & $1.62,1.72$ \\
\hline & Age, SES, EUR & 1.44 & $1.40,1.49$ & 1.67 & $1.62,1.71$ \\
\hline & Age, SES, AMI & 1.46 & $1.42,1.51$ & 1.68 & $1.63,1.72$ \\
\hline \multirow[t]{2}{*}{ AFA } & Age & 1.46 & $1.40,1.51$ & 1.64 & $1.58,1.70$ \\
\hline & Age, SES, AFR & 1.42 & $1.37,1.48$ & 1.63 & $1.58,1.69$ \\
\hline \multirow[t]{4}{*}{ HA } & Age & 1.61 & $1.52,1.71$ & 1.68 & $1.60,1.75$ \\
\hline & Age, SES, AFR & 1.55 & $1.46,1.65$ & 1.69 & $1.61,1.77$ \\
\hline & Age, SES, EUR & 1.55 & $1.46,1.65$ & 1.68 & $1.60,1.77$ \\
\hline & Age, SES, AMI & 1.56 & $1.47,1.65$ & 1.68 & $1.60,1.77$ \\
\hline
\end{tabular}

SES, BMI and WHR were divided by their SDs

${ }^{a}$ Groups are defined as noted in the footnote to Table 1

${ }^{\mathrm{b}}$ BMI or WHR was used as the covariate of interest in the models, adjusting for age (entry age), SES and each of the genetic admixtures: AFR, EUR or AMI separately

All $p$ values $<1.0 \times 10^{-40}$

positive association between AMI admixture and diabetes (OR 1.6), adjusting for age, sex, BMI and education, but it did not reach statistical significance $(95 \%$ CI $0.6,4.3)$, which might be due to the limited sample size [16].

In our study, AFR admixture was not significantly associated with diabetes risk among the HA women, which may be due to the rather low AFA contribution in this WHI population group. EUR admixture appeared to have a protective effect against diabetes risk in the Hispanic group, with and without adjusting for SES and/or BMI. The results are consistent with the findings from a recent case-control study by Florez et al. [15] for Latinos in Mexico, but are different from their findings for Latinos in Colombia. This study found that EUR admixture was associated with lower diabetes risk in Mexicans (OR 0.17, $p=0.02$ ), after adjusting for SES and BMI while the association was not significant in Colombians (OR $0.64, p=0.46$ ) [15]. Compared with these studies of Hispanics $[15,16]$, our study is population based, has a much larger sample size, is restricted to postmenopausal women living in the USA and also uses a different measure of SES.

The current study shows that while admixture associations remained significant the effect was smaller (decreased HR) after adjusting for SES. This is similar to previous studies in Hispanic population groups [21-24, 26, 27] that suggest that SES can confound the interpretation of admixture analyses. Although it is not possible to be sure that our or other measurements of SES capture all psychosocial confounders, we believe our study suggests that the contribution of the genetic admixture to diabetes risk is partially independent of these other factors. However, we also note 
that more of the explained randomness (analogous to explained variation) can be attributed to measures of adiposity and SES than admixture in AFA and HA women: BMI (12.7\% and $18.9 \%$, respectively), WHR $(21.3 \%$ and $26.3 \%$, respectively), SES (3.4\% and $9.6 \%$, respectively) and admixture $(1.8 \%$ and $2.3 \%$, respectively).

Our study has important limitations. In the current study, individuals were classified as diabetic and non-diabetic by self-reported treatment with glucose-lowering medications or physician diagnoses of diabetes in the absence of pregnancy. While glucose measurements or insulin/insulin resistance was not available for the current study, previous WHI studies have validated this method [43]. Additional studies will be necessary to determine if insulin resistance itself or diabetic endophenotypes are also associated with admixture. Another limitation is our use of entry data for assessing BMI, WHR and SES. Ideally, it would be useful to have longitudinal information for these factors (not available for the majority of these participants) to more accurately assess the effect of these variables as both risk factors and covariates in the models tested.

Consistent with previous studies [15], SES was inversely associated with diabetes risk in our study. Moreover, we also found that adjusting for admixture had little effect on this association. Unravelling the complex relationships of admixture, socioeconomic factors and type 2 diabetes is challenging. Low SES can increase diabetes risk via various mechanisms including poor access to healthcare, neglect of preventive strategies or unhealthy diet $[1,44]$. In populations where low SES is highly correlated to ancestral background, SES could be an intermediate factor on the pathway of admixture and diabetes.

Previous studies have shown elevated diabetes prevalence among AFAs and HAs compared with EAs, even after accounting for adiposity [1, 44-46]. Our results on genetic admixture are generally in accordance with these studies. Moreover, it is interesting to note in our study that in AFAs the HR for AFR admixture reduced from 2.36 (when adjusting for entry age and SES) to 2.02 when further adjusting for $\mathrm{BMI}$, while it had smaller reduction (to 2.23) when adjusting for WHR instead of BMI. In contrast, in the HA group, in which most participants have higher AMI admixture, the HRs for AMI admixture were almost the same before and after adjusting for BMI (1.97 vs 2.03) while the HR reduced to 1.42 and the association became non-significant when adjusting for WHR. This might be because AFR admixture is highly associated with BMI but has no association with WHR among AFA, while in HA, AMI admixture is significantly associated with WHR but not with BMI, as shown in our previous study of admixture and adiposity [19]. A possible explanation is that ethnic differences exist in visceral adipose tissue and, based on computed tomography scans, AFAs have less visceral fat than other self-reported ethnic groups [47]. Hence, although WHR is not correlated with AFR admixture among AFAs, WHR appears to be an important adiposity measure in populations with substantial AMI admixture. We believe it is likely that WHR is also a mediator of the admixture association with diabetes risk in HAs, which would in part account for the large effect of WHR in the AMI association model in HAs (Table 2).

We also investigated the association of adiposity with diabetes risk accounting for genetic admixture. Higher BMI was associated with a greater risk for diabetes in both AFA and HA women, before and after adjusting for genetic admixture and SES. Similar results were observed for WHR. Although longitudinal adiposity measurements prior to study entry (i.e. before the age of 50) were not available, the association of adiposity with diabetes risk suggests that adiposity has an independent contribution to the development of diabetes apart from SES and genetic admixture. We also considered both BMI and WHR together in the same analysis and the results suggest that each partially contributes independently to diabetes risk (ESM Table 3).

In conclusion, we found a significant association between AFR admixture and diabetes risk in AFA women and AMI admixture and diabetes risk in HA women. Our data are consistent with previous findings that even within a single population of mixed ancestry, disease risk can vary with admixture. Although we cannot exclude that other factors including insulin resistance and diet may underlie or interact with genetic differences, the data provide additional support to the hypothesis that differences in ethnic origins are critical to aetiological studies. Our results also suggest that admixture mapping may be a useful method for identifying loci underlying this complex disease and that admixture should also be considered in evaluating therapeutic intervention. In accord with previous studies, our data also show that SES and adiposity measures including BMI and WHR are important risk factors for diabetes development. Further controlling for genetic admixture did not change these associations. Thus, despite the complex interplay between genetic admixture, SES, BMI and WHR on diabetes, each of these independently attributes to diabetes risk after accounting for the other factors.

Acknowledgements We thank the participants of the WHI and acknowledge the contributions of WHI investigators for the development of study materials (see acknowledgements in the ESM). We thank the journal editor, the associate editor and the referees for their constructive comments, and also thank R. Prentice ( Fred Hutchinson Cancer Research Center, Seattle, WA, USA) and J. Rossouw ( National Heart, Lung, and Blood Institute, Bethesda, Washington DC, USA) for their support during the manuscript revision.

Funding The WHI programme is funded by the National Heart, Lung, and Blood Institute (NHLBI), National Institutes of Health, US Department of Health and Human Services through contracts HHSN268201100046C, HHSN268201100001C, HHSN268201100002C, HHSN268201100003C, HHSN268201100004C, and HHSN271201100004C. This work was supported by the National Institutes of Health NHLBI BAA contract number 
HHSN268200764319C. The study design was approved by the NHLBI as part of a BAA for the WHI. The WHI provided access to clinical data and DNA samples under appropriate institutional review board approval. The WHI Publication and Presentation Committee reviewed and approved the manuscript for submission. The NHLBI was not otherwise involved in the design and conduct of the study, or in the analysis of data or preparation of the manuscript.

Contribution statement LQ, RN, RK, LG, JR and MFS contributed to the conception and design of the study. All authors contributed to analysis and interpretation of the data. LQ and MFS drafted the first version of the manuscript. All authors critically revised the manuscript for important intellectual content and approved the final version for publication.

Duality of interest The authors declare that there is no duality of interest associated with this manuscript.

\section{References}

1. Maskarinec G, Grandinetti A, Matsuura G et al (2009) Diabetes prevalence and body mass index differ by ethnicity: the multiethnic cohort. Ethn Dis 19:49-55

2. Zimmet P, Alberti KG, Shaw J (2001) Global and societal implications of the diabetes epidemic. Nature 414:782-787

3. Centers for Disease Control and Prevention (2010) Health, United States, 2009 with Special Feature on Medical. Available from www.cdc.gov/nchs/data/hus/hus09.pdf, accessed 13 April 2011

4. Centers for Disease Control and Prevention (2011) National diabetes fact sheet: general information and national estimates on diabetes in the United States. Available from www.cdc.gov/diabetes/ pubs/pdf/ndfs_2011.pdf, accessed 13 April 2011

5. Fernandez JR, Shriver MD, Beasley TM et al (2003) Association of African genetic admixture with resting metabolic rate and obesity among women. Obes Res 11:904-911

6. Parra EJ, Marcini A, Akey J et al (1998) Estimating African American admixture proportions by use of population-specific alleles. Am J Hum Genet 63:1839-1851

7. Reiner AP, Carlson CS, Ziv E, Iribarren C, Jaquish CE, Nickerson DA (2007) Genetic ancestry, population sub-structure, and cardiovascular disease-related traits among African-American participants in the CARDIA Study. Hum Genet 121:565-575

8. Shaffer JR, Kammerer CM, Reich D et al (2007) Genetic markers for ancestry are correlated with body composition traits in older African Americans. Osteoporos Int 18:733-741

9. Tang H, Jorgenson E, Gadde M et al (2006) Racial admixture and its impact on BMI and blood pressure in African and Mexican Americans. Hum Genet 119:624-633

10. Bedoya G, Montoya P, Garcia J et al (2006) Admixture dynamics in Hispanics: a shift in the nuclear genetic ancestry of a South American population isolate. Proc Natl Acad Sci USA 103:7234-7239

11. Bertoni B, Budowle B, Sans M, Barton SA, Chakraborty R (2003) Admixture in Hispanics: distribution of ancestral population contributions in the Continental United States. Hum Biol 75:1-11

12. Bonilla C, Parra EJ, Pfaff CL et al (2004) Admixture in the Hispanics of the San Luis Valley, Colorado, and its implications for complex trait gene mapping. Ann Hum Genet 68:139-153

13. Klimentidis YC, Miller GF, Shriver MD (2009) The relationship between European genetic admixture and body composition among Hispanics and Native Americans. Am J Hum Biol 21:377-382

14. Chakraborty R, Ferrell RE, Stern MP, Haffner SM, Hazuda HP, Rosenthal M (1986) Relationship of prevalence of non-insulin- dependent diabetes mellitus to Amerindian admixture in the Mexican Americans of San Antonio, Texas. Genet Epidemiol 3:435-454

15. Florez JC, Price AL, Campbell D et al (2009) Strong association of socioeconomic status with genetic ancestry in Latinos: implications for admixture studies of type 2 diabetes. Diabetologia 52:1528-1536

16. Martinez-Marignac VL, Valladares A, Cameron E et al (2007) Admixture in Mexico City: implications for admixture mapping of type 2 diabetes genetic risk factors. Hum Genet 120:807-819

17. Parra EJ, Hoggart CJ, Bonilla C et al (2004) Relation of type 2 diabetes to individual admixture and candidate gene polymorphisms in the Hispanic American population of San Luis Valley, Colorado. J Med Genet 41:e116

18. Cheng CY, Reich D, Coresh J et al (2010) Admixture mapping of obesity-related traits in African Americans: the Atherosclerosis Risk in Communities (ARIC) Study. Obesity (Silver Spring) 18:563-572

19. Nassir R, Qi L, Kosoy R et al (2011) Relationship between adiposity and admixture in African-American and Hispanic-American women. Int J Obes. doi:10.1038/ijo.2011.84

20. Keene KL, Mychaleckyj JC, Leak TS et al (2008) Exploration of the utility of ancestry informative markers for genetic association studies of African Americans with type 2 diabetes and end stage renal disease. Hum Genet 124:147-154

21. LaVeist TA, Thorpe RJ Jr, Galarraga JE, Bower KM, Gary-Webb TL (2009) Environmental and socio-economic factors as contributors to racial disparities in diabetes prevalence. J Gen Intern Med 24:1144-1148

22. Link CL, McKinlay JB (2009) Disparities in the prevalence of diabetes: is it race/ethnicity or socioeconomic status? Results from the Boston Area Community Health (BACH) survey. Ethn Dis 19:288-292

23. Robbins JM, Vaccarino V, Zhang H, Kasl SV (2005) Socioeconomic status and diagnosed diabetes incidence. Diabetes Res Clin Pract 68:230-236

24. Signorello LB, Schlundt DG, Cohen SS et al (2007) Comparing diabetes prevalence between African Americans and Whites of similar socioeconomic status. Am J Public Health 97:2260-2267

25. Krishnan S, Cozier YC, Rosenberg L, Palmer JR (2010) Socioeconomic status and incidence of type 2 diabetes: results from the Black Women's Health Study. Am J Epidemiol 171:564-570

26. Robbins JM, Vaccarino V, Zhang H, Kasl SV (2000) Excess type 2 diabetes in African- American women and men aged 40-74 and socioeconomic status: evidence from the Third National Health and Nutrition Examination Survey. J Epidemiol Community Health 54:839-845

27. Robbins JM, Vaccarino V, Zhang H, Kasl SV (2001) Socioeconomic status and type 2 diabetes in African American and non-Hispanic white women and men: evidence from the Third National Health and Nutrition Examination Survey. Am J Public Health 91:76-83

28. de Koning L, Merchant AT, Pogue J, Anand SS (2007) Waist circumference and waist-to-hip ratio as predictors of cardiovascular events: meta-regression analysis of prospective studies. Eur Heart J 28:850-856

29. Escobedo J, Buitron LV, Velasco MF et al (2009) High prevalence of diabetes and impaired fasting glucose in urban Latin America: the CARMELA Study. Diabet Med 26:864-871

30. Zhang Q, Wang Y, Huang ES (2009) Changes in racial/ethnic disparities in the prevalence of type 2 diabetes by obesity level among US adults. Ethn Health 14:439-457

31. Zhu X, Luke A, Cooper RS et al (2005) Admixture mapping for hypertension loci with genome-scan markers. Nat Genet 37:177-181

32. Kosoy R, Nassir R, Tian C et al (2009) Ancestry informative marker sets for determining continental origin and admixture proportions in common populations in America. Hum Mutat 30:69-78

33. Nassir R, Kosoy R, Tian C et al (2009) An ancestry informative marker set for determining continental origin: validation and extension using human genome diversity panels. BMC Genet 10:39 
34. The Women's Health Initiative Study Group (1998) Design of the Women's Health Initiative clinical trial and observational study. Control Clin Trials 19:61-109

35. Hays J, Hunt JR, Hubbell FA et al (2003) The Women's Health Initiative recruitment methods and results. Ann Epidemiol 13:S18S77

36. Langer RD, White E, Lewis CE, Kotchen JM, Hendrix SL, Trevisan M (2003) The Women's Health Initiative Observational Study: baseline characteristics of participants and reliability of baseline measures. Ann Epidemiol 13:S107-S121

37. Falush D, Stephens M, Pritchard JK (2003) Inference of population structure using multilocus genotype data: linked loci and correlated allele frequencies. Genetics 164:1567-1587

38. Pritchard JK, Stephens M, Donnelly P (2000) Inference of population structure using multilocus genotype data. Genetics 155:945-959

39. Whitsel EA, Quibrera PM, Smith RL et al (2006) Accuracy of commercial geocoding: assessment and implications. Epidemiol Perspect Innov 3:8

40. Whitsel EA, Rose KM, Wood JL, Henley AC, Liao D, Heiss G (2004) Accuracy and repeatability of commercial geocoding. Am J Epidemiol 160:1023-1029
41. Cox DR (1973) Regression models and life tables (with discussion). J Royal Statist Soc B 34:187-220

42. O'Quigley J, Xu R, Stare J (2005) Explained randomness in proportional hazards models. Stat Med 24:479-489

43. Margolis KL, Lihong Q, Brzyski R et al (2008) Validity of diabetes self-reports in the Women's Health Initiative: comparison with medication inventories and fasting glucose measurements. Clin Trials 5:240-247

44. Shai I, Jiang R, Manson JE et al (2006) Ethnicity, obesity, and risk of type 2 diabetes in women: a 20 -year follow-up study. Diabetes Care 29:1585-1590

45. Bermudez OI, Tucker KL (2001) Total and central obesity among elderly Hispanics and the association with type 2 diabetes. Obes Res 9:443-451

46. Stern MP, Gaskill SP, Hazuda HP, Gardner LI, Haffner SM (1983) Does obesity explain excess prevalence of diabetes among Mexican Americans? Results of the San Antonio Heart Study. Diabetologia 24:272-277

47. Araneta MR, Barrett-Connor E (2005) Ethnic differences in visceral adipose tissue and type 2 diabetes: Filipino, AfricanAmerican, and white women. Obes Res 13:1458-1465 